\title{
Pathological Features of Ruptured Coronary Plaque and Thrombus Interfaces: Fibrin and von Willebrand Factor as Platelet Scaffolds on Rupture Sites
}

\author{
Atsushi Yamashita ${ }^{1}$ Kensaku Nishihira ${ }^{1,2}$ Toshihiro Gi ${ }^{1}$ Kazunari Maekawa ${ }^{1}$ Kinta Hatakeyama ${ }^{3,4}$ \\ Saki Horiuchi ${ }^{1}$ Kei Wada ${ }^{5}$ Yoshisato Shibata ${ }^{2}$ Yujiro Asada ${ }^{1}$
}

${ }^{1}$ Department of Pathology, Faculty of Medicine, University of Miyazaki, Miyazaki, Japan

2 Department of Cardiology, Miyazaki Medical Association Hospital, Miyazaki, Japan

${ }^{3}$ Department of Diagnostic Pathology, Nara Medical University, Kashihara, Japan

${ }^{4}$ Department of Pathology, National Cerebral and Cardiovascular Center, Osaka, Japan

${ }^{5}$ Department of Medical Sciences, Faculty of Medicine, University of Miyazaki, Miyazaki, Japan

Thromb Haemost 2021;121:234-241.
Address for correspondence Atsushi Yamashita, MD, Department of Pathology, Faculty of Medicine, University of Miyazaki, 5200 Kihara, Kiyotake, Miyazaki 889-1692, Japan

(e-mail: atsushi_yamashita@med.miyazaki-u.ac.jp).

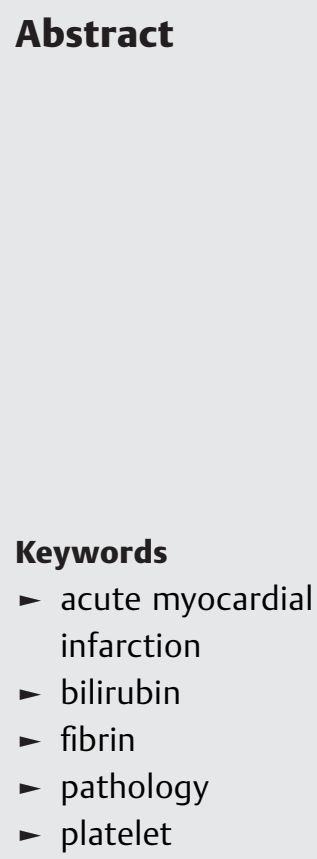

Arterial thrombus formation is thought to be initiated by platelet adhesion to the subendothelial matrix, but ruptured atherosclerotic plaques are characterized by substantial reduction of matrix proteins compared with stable plaques. Intraplaque erythrocytes and/or fibrin have been reported in high-risk coronary plaques. The aims of the current study were to identify factors that provide scaffolds for platelets at the sites of ruptured coronary plaques and investigate depositions of iron and bilirubin as hemoglobin catabolites in the ruptured plaques. Histological characteristics of plaque components and the thrombus interface were examined in 73 acute coronary aspirated thrombi. Necrotic debris (95\%), macrophages (95\%), and cholesterin clefts (81\%) were observed frequently at the ruptured plaque and thrombus interface. A fibrous matrix (47\%), calcification (32\%), and extracellular deoxyribonucleic acid (15\%) were identified as small foci. Tissue factor was localized in the necrotic core and macrophages. Fibrin and von Willebrand factor were consistently deposited within the plaques and beneath platelet aggregations. The citrullinated histone H3-immunopositive area accounted for only $0.5 \%$ of the plaque area. Bilirubin and iron depositions were detected in approximately $20 \%$ of the plaques in addition to biliverdin reductase and ferritin expression in macrophages. Fibrin and von Willebrand factor rather than matrix proteins and neutrophil extracellular traps may be major adhesive molecules at the sites of ruptured plaques. Iron and bilirubin deposits may be markers for rupture-prone plaques.

\section{Introduction}

Acute myocardial infarction (AMI) is triggered by coronary atherosclerotic plaque disruption and subsequent formation of large thrombus. It is generally thought that arterial throm- bus formation is initiated by platelet adhesion to the exposed subendothelial collagenous matrix via von Willebrand factor (VWF). ${ }^{1}$ Plaque rupture, a morphologic feature of plaque disruption, accounts for 55 to $65 \%$ of cases of acute coronary thrombosis. ${ }^{2}$ Ruptured plaques are characterized by the received

June 2, 2020

accepted after revision

July 26, 2020

published online

September 13, 2020
DOI https://doi.org/

$10.1055 / \mathrm{s}-0040-1716539$.

ISSN 0340-6245.

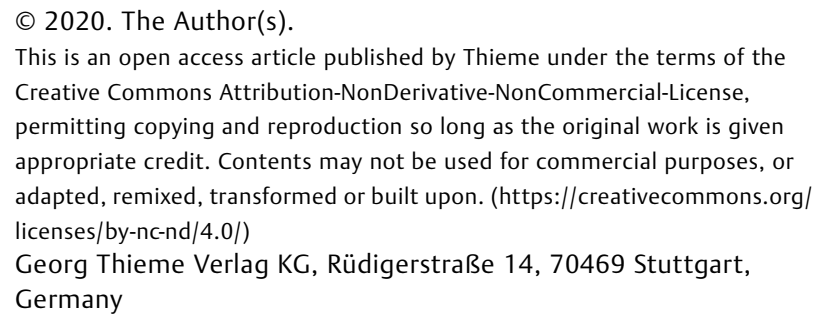


presence of a large necrotic core and substantial reduction of fibrous matrix by the effects of matrix metalloproteinases compared with stable plaques. ${ }^{3}$ The necrotized core rather than the fibrous matrix is exposed to the luminal surface at plaque rupture. ${ }^{3}$ Therefore, there is a significant discrepancy between the current concept of arterial thrombus formation and pathological findings of ruptured plaques. The findings suggest the presence of platelet scaffolds other than the fibrous matrix in the necrotized core elements.

Neutrophil extracellular traps (NETs), fibrin, and VWF are candidate scaffolds for the adhesion of platelets. NETs are networks of deoxyribonucleic acid (DNA) fibers consisting of histones and antimicrobial proteins released from neutrophils. NETs perfused with blood promote platelet adhesion, activation, and aggregation, and recruit erythrocytes. ${ }^{4,5}$ Pathological studies have detected NETs in coronary atherothrombi as well as thrombotic and hemorrhagic coronary plaques ${ }^{6,7}$ Fibrin can be generated after plaque rupture, ${ }^{8}$ plasma leakage, ${ }^{9}$ and intraplaque hemorrhage ${ }^{10}$ in the advanced atherosclerotic plaques. VWF is consistently present in aspirated coronary thrombi in patients with AMI. ${ }^{11}$ However, the presence of NETs, fibrin, and VWF on the surface of the ruptured plaques has not been investigated in patients with AMI.

Clinical studies suggest that intraplaque hemorrhage accelerates plaque progression via accumulation of free cholesterol from erythrocyte membranes. ${ }^{12,13}$ We recently reported that a high event risk finding of magnetic resonance imaging reflects intraplaque hemorrhage, deposition of erythrocytes, and fibrin. ${ }^{10}$ Hemoglobin released from erythrocytes is catabolized to iron and bilirubin in macrophages. ${ }^{14}$ The findings suggest presence of hemoglobin catabolites in the ruptured plaques. Notably, however, iron and bilirubin depositions have not been thoroughly investigated in ruptured coronary plaques of AMI patients.

We hypothesized that platelet scaffolds in addition to collagen are present on ruptured plaques, and that intraplaque hemorrhage often precedes plaque rupture. In the present study, we investigated factors that potentially contribute to platelet scaffolds at the sites of ruptured coronary plaques as well as iron and bilirubin depositions in ruptured plaques of AMI patients within 24 hours of onset.

\section{Methods}

\section{Patient Population}

The present study examined thrombi removed from 287 consecutive cases via an aspiration catheter (Thrombuster, Kaneka Co., Tokyo, Japan) within 24 hours of AMI onset between January 2007 and August 2012. The ethics committees of the participating institutions approved the study protocol (0-0224).

\section{Tissue Processing}

Thrombi aspirated from the 287 patients via an aspiration catheter were fixed immediately in $4 \%$ paraformaldehyde and embedded in paraffin for histological evaluation. Fourmicrometer-thick sections were stained with hematoxylin and eosin. Disrupted plaque components with thrombus, indicating plaque rupture, were detected in $86 / 287$ samples. Based on the histological findings, we considered the disrupted plaques with thrombus as ruptured plaques. After exclusion of 13 samples with small $\left(<1 \mathrm{~mm}^{2}\right)$ atherosclerotic plaque components, the remaining 73 samples underwent morphological analysis by hematoxylin and eosin, AZAN trichrome, and Berlin blue staining.

\section{Histological Evaluation}

To examine platelet scaffolds at the sites of ruptured coronary plaques as well as iron and bilirubin depositions in the ruptured plaques, morphological parameters were assessed at the ruptured plaque and thrombus interface, including the presence or absence of necrotic debris, macrophage infiltration, a cholesterin cleft, calcification, hematoxylin-positive extracellular DNA, and an AZAN trichrome-positive fibrous matrix, or discolored fibrous matrix and yellow deposition (-Supplementary Fig. S1, available in the online version). Intracellular and extracellular iron depositions were assessed by Berlin blue staining, and bilirubin deposition was assessed by the Gmelin method. ${ }^{15}$ Two pathologists (A.Y. and T.G.) independently performed the histological analysis. There were no interobserver or intraobserver differences in necrotic debris, macrophage infiltration, or cholesterin cleft. The interobserver $\mathrm{k}$ coefficients of calcification, extracellular DNA, and a fibrous matrix, discolored fibrous matrix, yellow deposition, and intracellular and extracellular iron depositions were $0.899,0.587,0.786,0.563,0.724,0.378$, and 0.381 , respectively, and the intraobserver $\mathrm{k}$ coefficients of them were $0.908,0.588,0.764,0.702,0.908,0.588,0.714$, and 0.915 , respectively. Cases with disconcordance were reviewed together with a digital monitor connected to a microscope to reach consensus. ${ }^{16}$

\section{Immunohistochemistry}

To examine scaffold proteins at the sites of ruptured coronary plaques, cellular components, and hemoglobin catabolism in the ruptured plaques, 73 samples were stained immunohistochemically using antibodies against fibrin (mouse monoclonal, clone T2G1; Accurate Chemical and Scientific Corp., Westbury, New York, United States) and VWF (sheep polyclonal, The Binding Site, Birmingham, United Kingdom). Twenty-eight samples were stained immunohistochemically using antibodies against platelet glycoprotein IIb/IIIa (sheep polyclonal; Affinity Biologicals Inc., Hamilton, California, United States), macrophage marker CD68 (mouse monoclonal, clone PGM-1a; DAKO, Glostrup, Denmark), neutrophil marker CD66b (mouse monoclonal, clone 6/40c; BioLegend Inc., San Diego, California, United States), smooth muscle cell marker smooth muscle actin (mouse monoclonal, clone 1A4; DAKO), and NETs marker citrullinated histone H3 (Cit-H3) (rabbit polyclonal; Abcam, Cambridge, Massachusetts, United States). Ten samples were stained immunohistochemically using antibodies against tissue factor (TF) (mouse monoclonal, clone H-9; Santa Cruz Biotechnology, Inc., Dallas, Texas, United States), biliverdin reductase (rabbit polyclonal; Novus Biologicals, Littleton, Colorado, United States), and ferritin (rabbit monoclonal, clone EPR3005Y; Abcam). The negative controls included 
nonimmune mouse, rabbit, or sheep immunoglobulin G (Jackson ImmunoResearch, Baltimore, Massachusetts, United States). The sections were stained with EnVision anti-mouse or rabbit immunoglobulin (DAKO) or an anti-sheep secondary antibody (Jackson ImmunoResearch). Horseradish peroxidase activity was visualized using a 3,3'-diaminobenzidine solution containing hydrogen peroxide, and the sections were counterstained with Meyer's hematoxylin.

\section{Quantitative Methods}

Microscopy images of immunopositive areas were captured using image analysis software (NIS Element Version 5.01, Nikon, Tokyo, Japan). Areas of CD68, CD66b, smooth muscle actin, and Cit-H3 staining were semiquantified using a color image analysis system (WinRoof, Mitani, Fukui, Japan). These areas are expressed as ratios of positively stained areas per total plaque area or thrombus area, as described by Yamashita et al. ${ }^{17}$

\section{Statistical Analysis}

Data are expressed as medians and interquartile ranges or means \pm standard deviation. A $p$-value of $<0.05$ was deemed to indicate statistical significance.

\section{Results}

\section{Patient Characteristics}

The clinical characteristics of the 73 patients within 24 hours of AMI onset are shown in - Table 1. Their mean age was 67 years, and $75 \%$ were male and $25 \%$ were female. The vast majority (95\%) was diagnosed with ST-elevation myocardial infarction, $60 \%$ had hypertension, and $66 \%$ had dyslipidemia. The prior use of calcium channel blocker, angiotensin-converting enzyme inhibitor/angiotensin receptor blocker, antiplatelets/coagulant, and statin was $32,26,26$, and $14 \%$ of patients, respectively, upon admission. The antiplatelets/coagulants usage consisted of antiplatelets (aspirin with or without thienopyridine: 17; other: 1 ), and aspirin and warfarin (1). Most thrombi were aspirated from the right coronary artery or left anterior descending arteries.

\section{Pathological Findings at the Ruptured Plaque and Thrombus Interface}

To examine platelet scaffolds at the sites of ruptured coronary plaques, pathological findings and immunohistochemistry of platelets, TF, and fibrin were assessed at the ruptured plaque and thrombus interface. Necrotic debris, macrophage infiltrate, and cholesterin clefts were frequently observed at the interface. A blue-stained fibrous matrix was evident in approximately half of the interface, but as small fragments. Calcification was detected in $32 \%$ of the interfaces, and hematoxylin-positive extracellular DNA was detected in $16 \%$ of the interfaces (-Fig. 1 and - Table 2). Immunohistochemistry staining of disrupted plaques with thrombi is shown in -Fig. 2. All 28 samples exhibited platelet-rich thrombus formation on disrupted plaques, and fibrin deposition in the plaques. CD68 and TF staining revealed the presence of macrophages and expression of TF in the plaques. Fibrin deposition was observed around macrophages and cholesterin clefts, and in necrotic debris. In high magnification images, fibrin depo-
Table 1 Clinical background of patients on aspirated coronary thrombi with plaque components

\begin{tabular}{|l|l|}
\hline Variable & $\begin{array}{l}\text { Acute myocardial } \\
\text { infarction }(\boldsymbol{n}=73)\end{array}$ \\
\hline Age, y & $67 \pm 12$ \\
\hline Male & $55(75)$ \\
\hline Hypertension & $44(60)$ \\
\hline Dyslipidemia & $40(55)$ \\
\hline Diabetes mellitus & $30(41)$ \\
\hline Obesity & $14(19)$ \\
\hline Smoking & $27(37)$ \\
\hline Old myocardial infarction & $10(14)$ \\
\hline Medications on admission & \\
\hline Antiplatelets or anticoagulant & $19(26)$ \\
\hline B-blocker & $5(7)$ \\
\hline ACE inhibitor or ARB & $19(26)$ \\
\hline Calcium channel blocker & $23(32)$ \\
\hline Statins & $10(14)$ \\
\hline ST-elevation myocardial infarction & $69(95)$ \\
\hline Onset to reperfusion time, h & $5.9 \pm 5.2$ \\
\hline Infarct-related artery & $33(45)$ \\
\hline Left anterior descending & $3(4)$ \\
\hline Left circumflex & $37(51)$ \\
\hline Right coronary artery & $135 \pm 93$ \\
\hline Laboratory data on admission & $137 \pm 37$ \\
\hline Total cholesterol (mg/dL) & \\
\hline Low-density lipoprotein \\
cholesterol (mg/dL)
\end{tabular}

Abbreviations: ACE, angiotensin-converting enzyme; ARB, angiotensin receptor blocker.

Note: Data are expressed as $n(\%)$ or mean \pm standard deviation (SD).

sition was evident at the plaque and platelet thrombus interface, but a fibrous matrix was not (-Fig. 2A).

To examine whether VWF was involved at the ruptured sites, we performed immunohistochemistry of platelets, VWF, and fibrin. Immunostaining of glycoprotein IIb/IIIa, VWF, and fibrin revealed the presence of VWF in ruptured sites and thrombi as fibrin deposits ( $\mathbf{- F i g . ~ 2 B}$ ) in all 73 rupture sites for fibrin and 72 of 73 rupture sites for VWF (-Table 2 ).

\section{Cellular Components and Cit-H3 in Ruptured Plaques}

Areas in ruptured plaques immunopositive for macrophages, neutrophils, smooth muscle cells, and Cit-H3 are shown in - Table 3. Macrophages and neutrophils were major cellular components in disrupted plaques, whereas smooth muscle cells were scarce. Neutrophils were distributed within the 


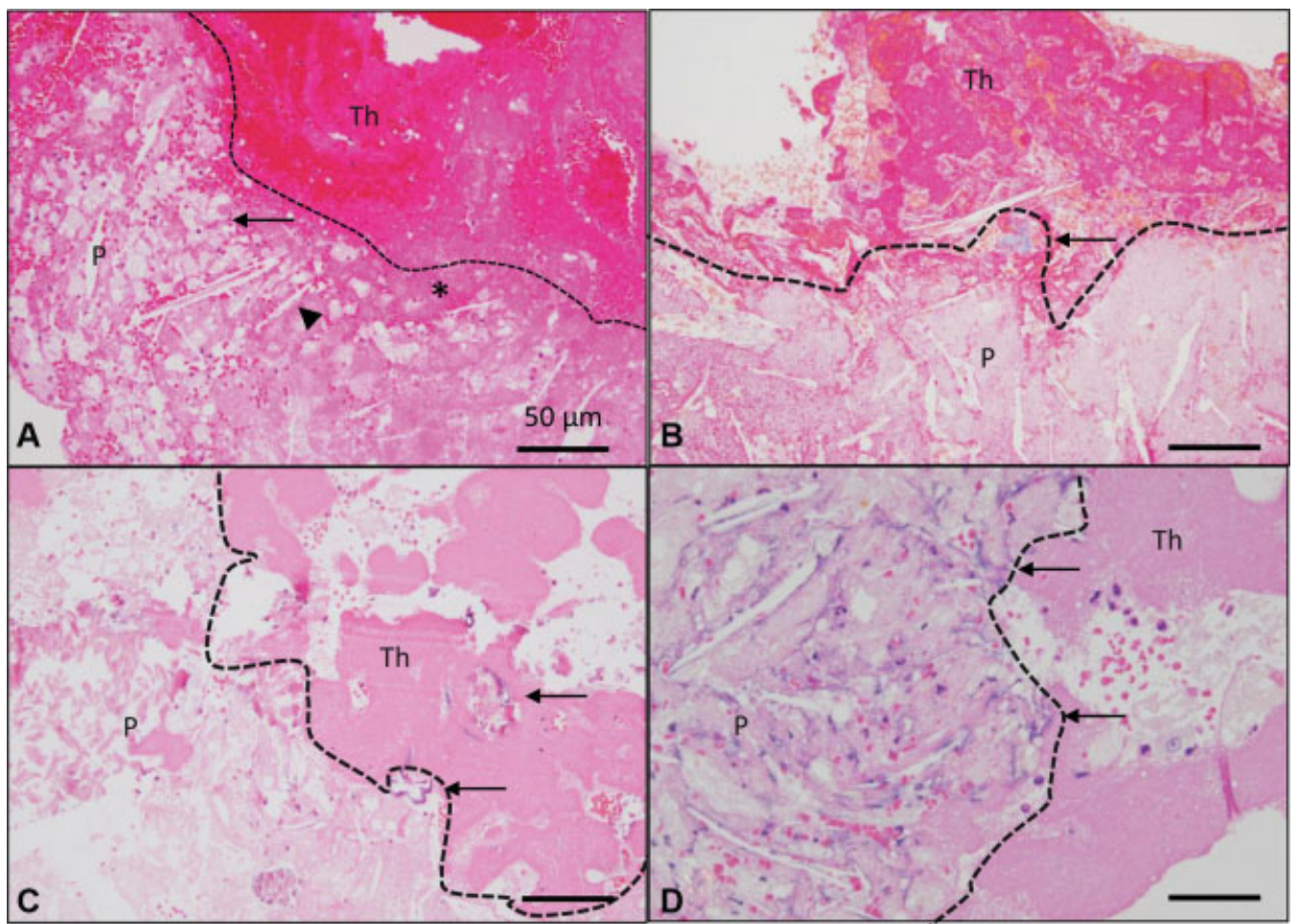

Fig. 1 Pathological findings at interfaces between ruptured plaques and thrombi in patients with acute myocardial infarction. Interfaces are indicated by dashed lines. (A) Necrotic debris (asterisk), macrophages (arrows), and cholesterin clefts (arrowhead) at the interface (hematoxylin and eosin stain). (B) Bluestained fragment of a fibrous matrix (arrow) at the interface (AZAN trichrome staining). (C) Calcification (arrows) at the interface (hematoxylin and eosin staining). (D) Basophilic extracellular deoxyribonucleic acid (DNA) (arrows) at the interface (hematoxylin and eosin staining). P, ruptured plaque; Th, thrombus.

Table 2 Pathological findings of ruptured plaques in patients with AMI

\begin{tabular}{|l|l|}
\hline Ruptured plaque and thrombus interface & Frequency (\%) \\
\hline Necrotic debris & 95 \\
\hline Macrophages & 95 \\
\hline Cholesterin clefts & 81 \\
\hline Fibrous matrix & 47 \\
\hline Calcification & 32 \\
\hline Extracellular DNA & 15 \\
\hline Fibrin & 100 \\
\hline von Willebrand factor & 99 \\
\hline Plaque & \\
\hline Yellow deposition & 23 \\
\hline Discolored fibrous matrix & 25 \\
\hline Intracellular iron deposition & 22 \\
\hline Extracellular iron deposition & 16 \\
\hline
\end{tabular}

Abbreviations: AMI, acute myocardial infarction; DNA, deoxyribonucleic acid. Note: $n=73$.

plaques and to accumulate around cholesterin clefts (-Fig. 2C). Immunoreactivity for Cit-H3 was observed in some neutrophil areas (-Fig. $\mathbf{2 C}$ ). In plaques, the median neutrophil-immunopositive area was $1.8 \%$ and the median Cit-H3-immunopositive area was $0.5 \%$. In coronary thrombi, the median Cit-H3-immunopositive area was $0.5 \%$ (interquartile range: $0.2-0.8 \%$ ).

\section{Discolored Fibrous Matrix, Yellow Deposits, and Iron Deposits}

Next, we examined pathological findings related to iron and bilirubin depositions in the ruptured plaques (-Table 2). A discolored fibrous matrix and yellow deposits were observed occasionally in the ruptured plaques. Representative images of yellow deposits, fragments of a discolored fibrous matrix, and intracellular and extracellular iron deposits are shown in - Fig. 3. Matrix and crystalloid materials were detected as yellow deposits ( $\mathbf{F i g}$. 3A). The deposits exhibited sequential color changes with oxidation as determined by the Gmelin method, which are used to detect bilirubin deposition histologically. The yellow color changed gradually to green, then blue, then light purple, and then disappeared ( - Fig. 3E and - Video 1). Most of the discolored fibrous matrixes were stained by Berlin blue, indicating iron deposition ( - Fig. 3B, D). Intracellular iron-positive cells were detected in $22 \%$ of the ruptured plaques. The appearance of intracellular iron was either dot-like or exhibited a diffuse cytoplasmic staining pattern (-Fig. 3C).

\section{Video 1}

Sequential color change of the yellow deposition with Gmelin method. The yellow color gradually changes to green, blue, and light purple, and finally disappears. Online content including video sequences viewable at: https://www.thieme-connect.com/products/ejournals/ html/10.1055/s-0040-1716539. 

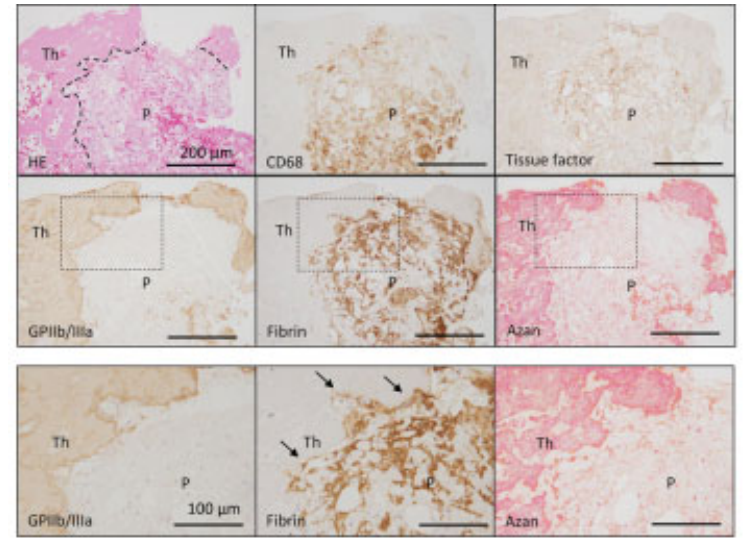

A
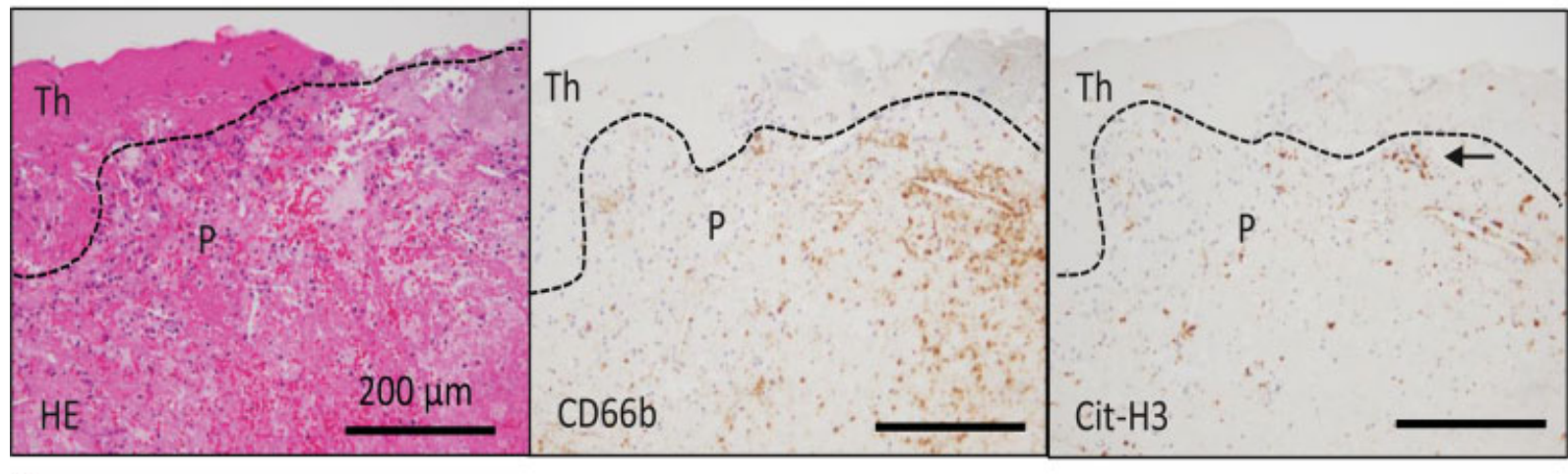

C

Fig. 2 Representative histological and immunohistochemical images of interfaces between ruptured plaques and thrombi in patients with acute myocardial infarction. Interfaces are indicated by dashed lines. (A) Immunohistochemical staining of CD68 indicating the presence of macrophages, and plaques stained positive for tissue factor. The thrombus on the ruptured plaque is rich in platelets, and some fibrin formation is visible in the thrombus (arrows). Fibrin deposition is evident around macrophages and cholesterin clefts, and in the necrotic core. In high magnification images (dashed squares), fibrin deposits are evident at the interface between the ruptured plaques and thrombus, and no fibrous matrix is observed in the AZAN-stained sample. (B) Immunohistochemical staining of glycoprotein IIb/IIla, von Willebrand factor, and fibrin revealed the presence of von Willebrand factor and fibrin at the interface. (C) Representative images depicting immunohistochemical staining of neutrophils (CD66b) and citrullinated histone $\mathrm{H3}$. Neutrophils are infiltrating the plaque and tended to accumulate around cholesterin clefts. Citrullinated histone $\mathrm{H} 3$ immunoreactivity is observed in some of the neutrophil area (arrow). Cit-H3, citrullinated histone H3; GPIIb/IIla, glycoprotein IIb/IIla; HE, hematoxylin and eosin; P, ruptured plaque; Th, thrombus; VWF, von Willebrand factor.

Table 3 Immunopositive areas in ruptured plaques in patients with AMI

\begin{tabular}{|l|l|}
\hline & Median (interquartile range) \% \\
\hline CD68 & $5.7(3.1-9.9)$ \\
\hline CD66b & $1.8(0.6-4.3)$ \\
\hline $\begin{array}{l}\text { smooth } \\
\text { muscle actin }\end{array}$ & $0(0-0.06)$ \\
\hline $\begin{array}{l}\text { Citrullinated } \\
\text { histone H3 }\end{array}$ & $0.5(0.05-1.43)$ \\
\hline
\end{tabular}

Abbreviation: AMI, acute myocardial infarction.

Note: $n=28$.

To examine the hemoglobin catabolic pathway, we performed immunohistochemistry of biliverdin reductase and ferritin. Immunohistochemical staining for biliverdin reductase and ferritin in ruptured plaques revealed accumulations of macrophages exhibiting various cytoplasmic colors ( - Fig. 3F). Ferritin and biliverdin reductase were expressed predominantly in yellow and gray cytoplasmic macrophages, but they were not expressed in all macrophages in ruptured plaques.

\section{Discussion}

Aspiration of acute coronary thrombi with plaque components facilitates examination of their cellular and molecular components without therapeutic or postmortem effects. The traditional view of arterial thrombus formation is initiation by platelet adhesion to the subendothelial matrix. ${ }^{1}$ The pathological findings at the interface and smooth muscle cell loss in the plaques are consistent with those of ruptured plaques in autopsied AMI patients. ${ }^{3}$ In a recent clinical study, optical coherence tomography-defined cholesterol crystals were detected at a high frequency $(81 \%)$ in coronary rupture sites of AMI patients. ${ }^{18}$ The plaque components at the thrombus included necrotic debris, macrophages, and cholesterin clefts, while a fibrous matrix was a minor 

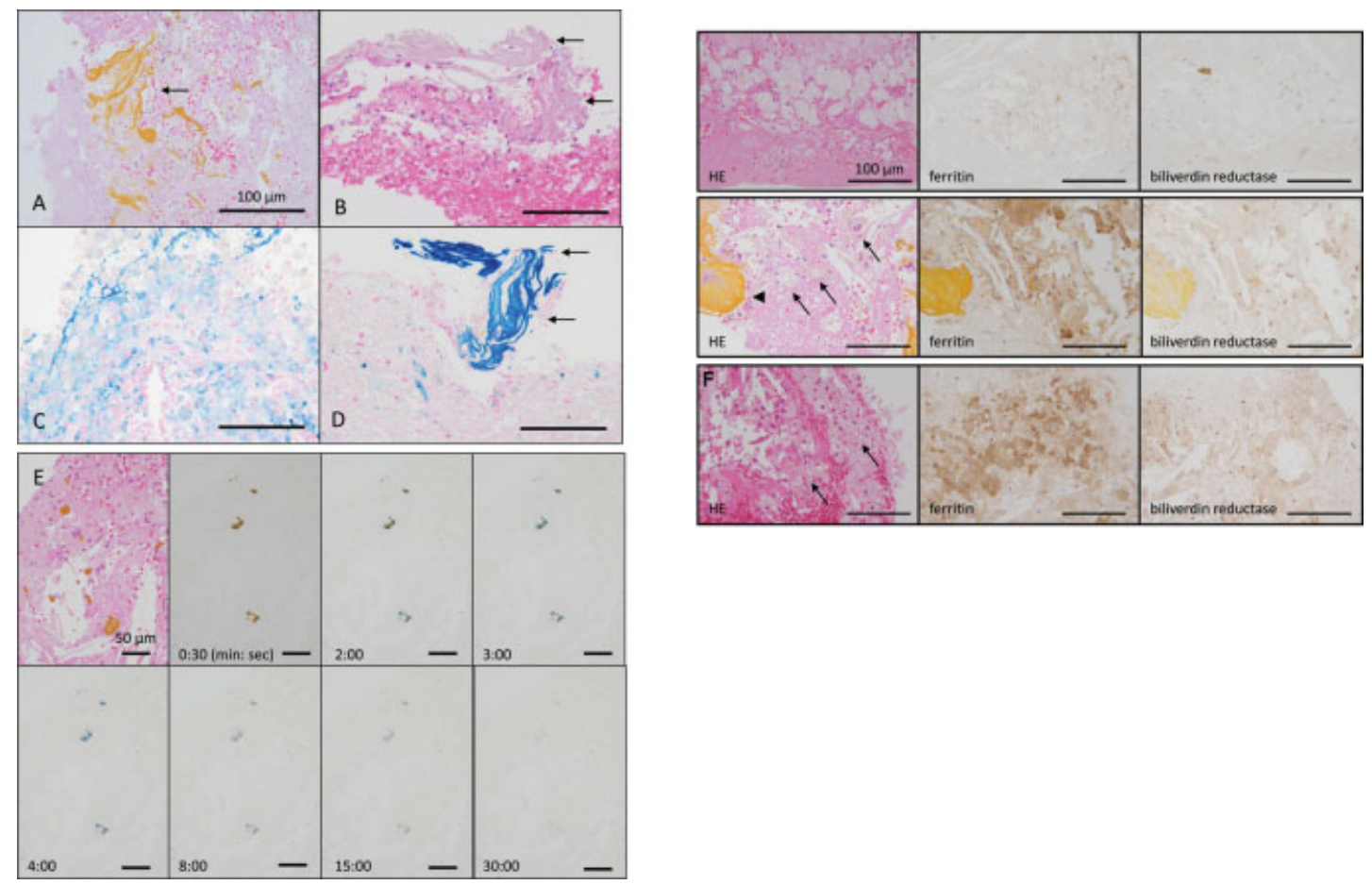

Fig. 3 Discolored fibrous matrix, yellow deposits, and iron deposits in ruptured plaques of patients with acute myocardial infarction. (A) Hematoxylin and eosin-stained sample depicting yellow deposits in a disrupted plaque (arrow). (B) Hematoxylin and eosin-stained sample depicting a fragment of a discolored fibrous matrix (arrows). (C) Berlin blue-stained sample in which intracellular iron is observed as dot-like or diffuse cytoplasmic staining patterns. (D) Berlin blue-stained sample depicting extracellular iron deposits corresponding to the discolored matrix in B. (E) Sequential color changes of yellow deposits assessed by the Gmelin method. The yellow color gradually changes to green, then blue, then light purple, and then disappears. (F) Histological images of macrophages and immunohistochemical images depicting staining of ferritin and biliverdin reductase at the ruptured plaque-thrombus interface. Ferritin and biliverdin reductase are expressed predominantly in yellowish (middle row, arrows) and grayish (bottom row, arrows) macrophages. Bilirubin deposition is present in the middle row (arrowhead). HE, hematoxylin and eosin.

\section{Coronary ruptured site and thrombus interface}

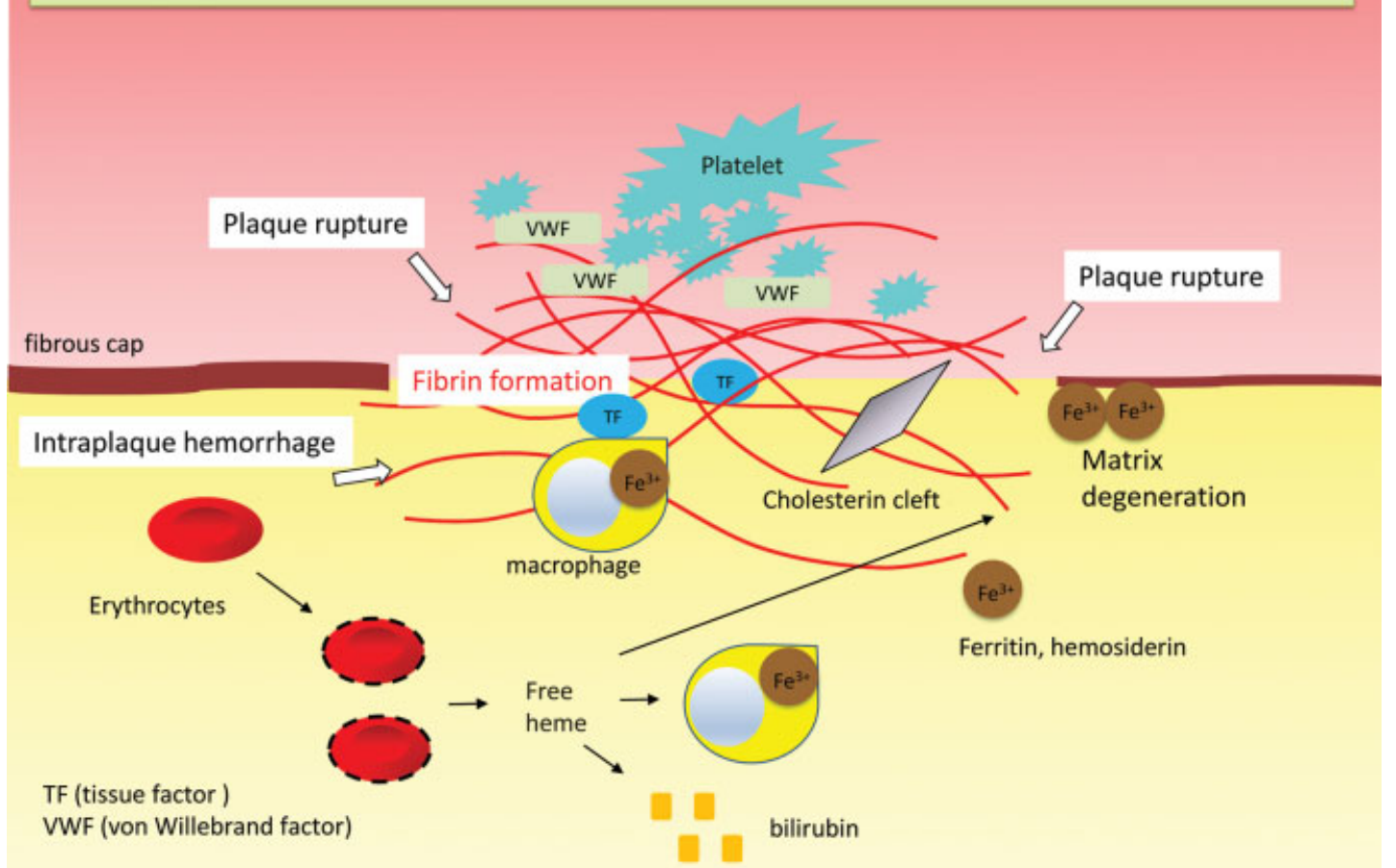

Fig. 4 Visual Summary: Coronary ruptured site and thrombus interface. 
component. However, platelets require adhesive molecules to bind exposed materials. TF in the necrotic debris and its expression in macrophages may contribute to fibrin formation at the plaque rupture. The consistent presence of fibrin and VWF at the ruptured sites supports the contention that fibrin and VWF are major adhesion molecules at the sites of ruptured plaques.

Pertiwi et $\mathrm{al}^{7}$ examined neutrophil infiltration and NET formation semiquantitatively in coronary plaques of autopsied AMI patients and control subjects. They concluded that neutrophils and NET formation were abundantly present in ruptured, eroded, and hemorrhagic plaques, but not in intact plaques. However, in their study, the respective median scores for neutrophil infiltration and NET formation in the ruptured plaques were 2 (multifocal positive cells in $>50 \%$ of the tissue area) and 1 (focal or few scattered positive cells in $<25 \%$ of the tissue area). The neutrophils and NET values are comparable with those of the present study. Extracellular DNA and NET formation are evidently not abundant on ruptured plaques.

Intraplaque hemorrhage is a feature of an advanced atherosclerotic plaque. Coronary thin-cap fibroatheroma, a rupture-prone plaque, had greater scores for intraplaque hemorrhage compared with early atherosclerotic lesions. ${ }^{12}$ Intracellular iron deposition was higher in coronary plaques of patients with unstable angina pectoris than that in patients with stable angina. ${ }^{19}$ The coronary hemorrhagic plaques were associated with increased expression of matrix metalloproteinase- 9 and TF. ${ }^{10}$ These pathological findings suggest that detection of an intraplaque hemorrhage is clinically important for risk stratification of patients with coronary atherosclerosis. The iron depositions in the discolored fibrous matrix and bilirubin deposition are novel findings of human coronary ruptured plaques. The yellowish or grayish macrophages expressed ferritin and biliverdin reductase in the plaques. Therefore, the iron and bilirubin depositions may have been derived from intraplaque heme metabolism after the hemorrhage. These deposits may be pathological features reflecting a previous intraplaque hemorrhage.

The current study had several limitations. First, we did not analyze whole disrupted plaques because we used the aspirated samples. It is therefore possible that the incidence of the detected pathological entities was underestimated and the extents of the cellular and molecular components were underor overestimated. Second, based on the histological findings, we considered disrupted plaques with a thrombus as ruptured plaques. Because we did not always confirm this by intravascular ultrasound or optical coherence tomography, we cannot deny the possibility of a coronary embolism. Third, this study excluded cases at $>24$ hours of AMI onset and cases of an aspirated thrombus with a small plaque component. Moreover, we did not use a method to reduce selection bias. Finally, prior use of medication can affect serum and plasma inflammatory and thrombus markers, ${ }^{20,21}$ as well as the pathological findings. However, the number of prior use of medication was too small to perform the contingency analysis.

In conclusion, fibrin and VWF rather than matrix proteins and NETs may be major adhesive molecules on coronary ruptured plaques. Iron and bilirubin depositions suggest a previous intraplaque hemorrhage and are potential markers of rupture-prone plaques ( - Fig. 4). The present study may provide insights into the mechanisms of thrombus formation on ruptured atherosclerotic plaques and risk stratification of patients with coronary atherosclerosis.

\section{What is known about this topic?}

- Arterial thrombus formation is initiated by platelet adhesion to the subendothelial matrix, but ruptured coronary plaques are characterized by substantial reduction of matrix proteins compared with stable plaques.

- Intraplaque hemorrhage may precede coronary plaque rupture.

\section{What does this paper add?}

- Fibrin and von Willebrand factor, but not collagen or neutrophil extracellular traps, on ruptured sites are major adhesive molecules.

- There are deposits of hemorrhage-related iron and bilirubin in the ruptured plaques.

\section{Funding}

This study was supported in part by Grants-in-Aid for Scientific Research in Japan (Numbers 19H03445, 16H05163, 16K08670, 19K07437, and 18K08123) from the Japan Society for the Promotion of Science, and the Cooperative Research Project Program of the Joint Usage/ Research Center at the Institute of Development, Aging and Cancer, Tohoku University.

\section{Conflict of Interest \\ None declared.}

\section{Acknowledgments}

We thank Ritsuko Sotomura, Kyoko Ohashi, Nahoko Udatsu, Yihan Zhang, Zeyu Liu, and Sun Jiaqi for excellent technical assistance with the immunohistochemistry. We also thank Dr. Owen Proudfoot and Mitchell Arico from Edanz Group (https://en-author-services.edanzgroup. $\mathrm{com} /$ ) for editing a draft of this manuscript.

\section{References}

1 Furie B, Furie BC. Mechanisms of thrombus formation. N Engl J Med 2008;359(09):938-949

2 Otsuka F, Yasuda S, Noguchi T, Ishibashi-Ueda H. Pathology of coronary atherosclerosis and thrombosis. Cardiovasc Diagn Ther 2016;6(04):396-408

3 Virmani R, Kolodgie FD, Burke AP, Farb A, Schwartz SM. Lessons from sudden coronary death: a comprehensive morphological classification scheme for atherosclerotic lesions. Arterioscler Thromb Vasc Biol 2000;20(05):1262-1275

4 Stakos D, Skendros P, Konstantinides S, Ritis K. Traps N' Clots: NETmediated thrombosis and related diseases. Thromb Haemost 2020;120(03):373-383 
5 Fuchs TA, Brill A, Duerschmied D, et al. Extracellular DNA traps promote thrombosis. Proc Natl Acad Sci U S A 2010;107(36): $15880-15885$

6 de Boer OJ, Li X, Teeling P, et al. Neutrophils, neutrophil extracellular traps and interleukin-17 associate with the organisation of thrombi in acute myocardial infarction. Thromb Haemost 2013; 109(02):290-297

7 Pertiwi KR, van der Wal AC, Pabittei DR, et al. Neutrophil extracellular traps participate in all different types of thrombotic and haemorrhagic complications of coronary atherosclerosis. Thromb Haemost 2018;118(06):1078-1087

8 Sato Y, Hatakeyama K, Yamashita A, Marutsuka K, Sumiyoshi A, Asada Y. Proportion of fibrin and platelets differs in thrombi on ruptured and eroded coronary atherosclerotic plaques in humans. Heart 2005;91(04):526-530

9 Tavora F, Cresswell N, Li L, Ripple M, Burke A. Immunolocalisation of fibrin in coronary atherosclerosis: implications for necrotic core development. Pathology 2010;42(01):15-22

10 Kuroiwa Y, Uchida A, Yamashita A, et al. Coronary high-signalintensity plaques on $\mathrm{T}_{1}$-weighted magnetic resonance imaging reflect intraplaque hemorrhage. Cardiovasc Pathol 2019; 40:24-31

11 Yamashita A, Sumi T, Goto S, et al. Detection of von Willebrand factor and tissue factor in platelets-fibrin rich coronary thrombi in acute myocardial infarction. Am J Cardiol 2006; 97(01):26-28

12 Kolodgie FD, Gold HK, Burke AP, et al. Intraplaque hemorrhage and progression of coronary atheroma. N Engl J Med 2003;349(24): 2316-2325

13 Takaya N, Yuan C, Chu B, et al. Presence of intraplaque hemorrhage stimulates progression of carotid atherosclerotic plaques: a high-resolution magnetic resonance imaging study. Circulation 2005; 111(21):2768-2775

14 Pimstone NR, Tenhunen R, Seitz PT, Marver HS, Schmid R. The enzymatic degradation of hemoglobin to bile pigments by macrophages. J Exp Med 1971;133(06):1264-1281

15 Orchard GE. Pigments and minerals. In: Suvarna SK, Layton C, Bancroft JD, eds. . Theory and Practice of Histological Techniques. 7th ed. Amsterdam: Elsevier; 2013:239-270

16 Dhall D, Kim SA, Mc Phaul C, et al. Heterogeneity of fibrosis in liver biopsies of patients with heart failure undergoing heart transplant evaluation. Am J Surg Pathol 2018;42(12):1617-1624

17 Yamashita A, Nishihira K, Matsuura Y, et al. Paucity of CD34positive cells and increased expression of high-mobility group box 1 in coronary thrombus with type 2 diabetes mellitus. Atherosclerosis 2012;224(02):511-514

18 Katayama Y, Tanaka A, Taruya A, et al. Feasibility and clinical significance of in vivo cholesterol crystal detection using optical coherence tomography. Arterioscler Thromb Vasc Biol 2020;40 (01):220-229

19 Nishihira K, Yamashita A, Imamura T, et al. Thioredoxin in coronary culprit lesions: possible relationship to oxidative stress and intraplaque hemorrhage. Atherosclerosis 2008;201(02):360-367

20 Sahebkar A, Serban C, Ursoniu S, et al; Lipid and Blood Pressure Meta-analysis Collaboration (LBPMC) Group. The impact of statin therapy on plasma levels of von Willebrand factor antigen. Systematic review and meta-analysis of randomised placebocontrolled trials. Thromb Haemost 2016;115(03):520-532

21 Liakos CI, Vyssoulis GP, Michaelides AP, et al. The effects of angiotensin receptor blockers vs. calcium channel blockers on the acute exercise-induced inflammatory and thrombotic response. Hypertens Res 2012;35(12):1193-1200 